\title{
Research on the Current Situation and Intervention of College Students 'Consciousness of Physical Exercise
}

\author{
Wanghaiyan ${ }^{1, *}$ \\ ${ }^{1}$ Sports Department of Nanjing Vocational College of Science and Technology, Nanjing, Jiangsu, China \\ *271640767@qq.com
}

\begin{abstract}
Although the national physical fitness test standard has been lowered three times, the analysis of the national physical fitness test data over the years shows that the physical condition of college students is gradually declining, and the physical fitness test data is not optimistic. The results of students' physical fitness test are not satisfactory, which is the inevitable result of students' low subjective demand and low participation in sports activities. Based on the questionnaire survey, this paper has a preliminary understanding of the current situation of College Students' awareness of physical exercise. According to the characteristics of the formation of sports awareness, appropriate intervention is carried out to guide students to form a good sense of physical exercise.
\end{abstract}

Keywords: physical exercise, consciousness, intervention

\section{大学生体育锻炼意识现状及其干预研究}

\author{
王海燕 ${ }^{1, a}$
}

${ }^{1}$ 南京科技职业学院体育部，南京，江苏，中国

${ }^{a} 271640767 @ q q . c o m$

\section{摘要}

尽管国家体质测试标准被下调三次，但是对国家体质测试多年来的数据分析发现，高校学生的体能状 况逐渐下降, 体质测试数据不容乐观。在校学生的体质测试成绩不尽如人意, 这是学生的主观需求不 高, 体育活动参与度较低的必然结果。本文通过问卷调查, 初步了解大学生体育锻炼意识现状, 根据 体育意识形成的特点进行适当干预，引导学生形成良好的体育锻炼意识。

关键词：体育锻炼；意识；干预

\section{1. 前言}

学生利用课余时间锻炼的人较少, 每天的阳光跑和 课外活动需要派专人多方位监督, 一年一次的运动会需 要用奖励操行分的方式动员, 很多同学上体育必修课就 是为了拿学分、或者因为是学校规定的课程等, 这都是 学生体育锻炼意识淡漠、体育参与的意识也较低的表现。 学生体育锻炼意识淡漠, 体育参与的意识也较低。本文 试图通过分析目前学生的体育锻炼意识现状, 从体育意 识形成的规律着手, 在教学过程中着重培养学生对运动 的兴趣, 激发学生培养体育锻炼意识, 养成锻炼的习惯, 提高身体素质。

\section{2. 背景和目的}

目前社会上体育活动开展相对丰富，民众的参与度 较高, 例如每年江苏范围内的马拉松赛事就多达几十个, 参与人次数十万。相比于社会上的体育赛事和活动, 大 学校园拥有较丰富、集中的体育资源和设施, 但学生参 与体育锻炼的情况不如普通群众。大学阶段作为连接学 校与社会的桥梁, 是学生进入社会之前接受系统体育教 育的最后时期, 是最终形成体育锻炼意识的重要阶段 ${ }^{[1]}$ 。 据调查发现目前大学生的课余时间, 基本都是用来上网、 打游戏或玩手机。只有很少同学能够利用课余时间主动 进行体育锻炼。很多同学长期养成一些不良习惯, 如抽 烟、謷夜、不吃早饭等。虽然知道身体健康很重要, 也 知道体育锻炼有利于增进身体健康, 但很多学生不愿意 安排时间主动参加体育锻炼 ${ }^{[2]}$ 。

体育科学词典中对体育意识的定义: 体育意识是运 动的现象、本质和规律在人们头脑中的反映, 是人们关 于体育运动的认知、情感、意志、价值观念和思想等统 
一的心理活动和认识系统 ${ }^{[3]}$ 。因此, 对大学生参与体育 锻炼的意识分析非常重要, 同时进行必要的干预有利于 运动现象和本质的形成, 通过适当的引导加强学生对体 育运动的认知和情感认同, 养成健全的体育锻炼意识。 从体育意识的根本上解决学生选择什么项目锻炼, 如何 坚持锻炼的问题。

本研究以南京科技职业学院大一、大二俱乐部学生 为调查对象, 着重对其体育锻炼的初级意识进行研究分 析, 通过调查和干预, 将学生目前对体育的兴趣、爱好、 情感; 体育运动的认知、情感 ${ }^{[4]}$; 以及对体育锻炼的科 学性和系统性的把握情况, 进行分析。通过研究, 了解 目前大学生体育锻炼意识现状。对不同体育意识层次, 进行有针对性的体育锻炼指导。着重解决好两个“引导”, 一是对还没养成体育锻炼意识的学生进行体育引导, 二 是对具有初级体育意识层次的学生向中级层次的转换 的引导。

\section{3. 调查内容}

本次问卷调查针对大一、大二俱乐部学生, 共十二 个问题，涉及五个方面的问题。

\section{1. 体育俱乐部的开设和场地设施方面}

如图 1 南京科技职业学院学生对目前开设体育俱乐 部模式的体育项目非常满意和比较满意的占 $78 \%$, 一般 的占 $22 \%$, 不满意为 0 。这一数据, 整体上可以反应学 生对体育俱乐部项目的设置和教学内容比较满意。说明 目前开设的大学课程在内容和形式上, 基本能满足学生 对体

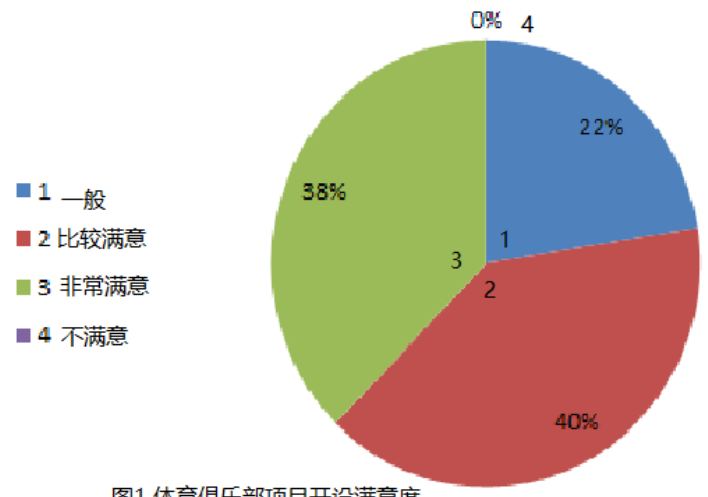

图1 体育俱乐部项目开设满意度

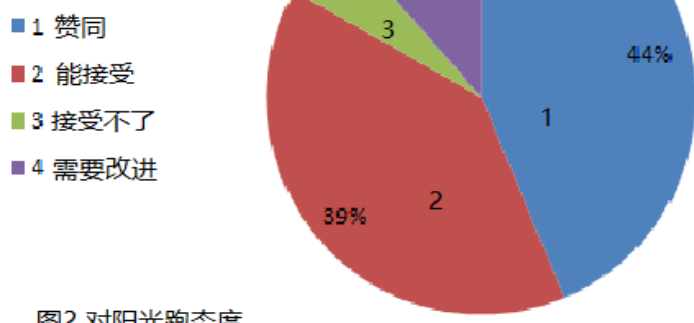

育学习的需要。对课外俱乐部的实施方面有 13. $9 \%$ 的同 学认为需要改进, $2.6 \%$ 的同学接受不了。具体改进方面 因为各个俱乐部情况不同, 学生分别提出了相应的改进 要求。对学校要求学生进行课外阳光跑步这一规定, 有 $44 \%$ 的同学表示赞同，39\%的表示能勉强接受，12\%的同 学提出需要改进。在体育场地设施满意度调查中满意度 达到 98\%，说明体育场地和设施的建立和使用上能基本 满足学生课内外的体育活动需要。

\section{2. 体育活动的参与度和获得感方面}

本次调查中发现, 每周平均参加课外体育活动的次 数达四次以上占 $18.7 \%, 2-3$ 次的占 $56.7 \%$, 还有 $1.6 \%$ 的同学从不进行课外体育活动。半数以上学生基本维持 在一周 2-3 次, 体育课外活动要求一学期 30 次合格, 45 次满分, 15 周时间, 也就意味着活动基本维持在合 格的次数上。在体育活动中能否获得成功体验的调查中 发现, 经常和偶尔获得成功体验的学生分别为 $42.2 \%$ 和 $43.2 \%, 14.6 \%$ 的学生能够一直获得成功体验。69.5\%的 同学能够偶尔与同伴或老师一起探讨运动技能和知识, 只有 $5.3 \%$ 的同学会一直这样探讨, 还有 $6.8 \%$ 的同学从 不和别人探讨这方面知识，属于埋头自己锻炼的人群。 有 $81.7 \%$ 学生希望在自主合作学习中, 有专业人士给予 指导, $16.2 \%$ 的人抱着无所谓的态度。从这类数据可以 分析, $85.5 \%$ 的学生有成功体验, $69.5 \%$ 的学生希望有人 一起探讨体育方面的相关知识, 表现出对所从事的体育 活动有进一步了解和感兴趣的趋势。

\section{3. 上体育课的动机和目的方面}

\section{表 1 上体育课目的和动机}

\begin{tabular}{|l|l|l|}
\hline 目的和动机 & 人 & 百分比 \\
\hline 为了考试 & 42 & $21.8 \%$ \\
\hline 学会科学地锻炼身体, 增强体能 & 161 & $85 \%$ \\
\hline 养成坚持锻炼的习惯 & 113 & $58.6 \%$ \\
\hline 能有机会和同学进行交流与合作 & 81 & $42.2 \%$ \\
\hline 学会运动技术并能运用 & 60 & $31.2 \%$ \\
\hline 满足运动兴趣和爱好 & 68 & $35.4 \%$ \\
\hline
\end{tabular}




\begin{tabular}{|l|l|l|}
\hline 获得一些健康相关的基本知识 & 62 & $32 \%$ \\
\hline 为了以后参与体育活动打基础 & 36 & $18.8 \%$ \\
\hline
\end{tabular}

如表 $185 \%$ 的学生为了学会科学锻炼, $58.6 \%$ 为了 养成体育锻炼的习惯。这部分学生上课动机明确, 意识 到需要养成科学锻炼的习惯。 $42.2 \%$ 能有机会与同学交 流, $35.4 \%$ 的学生为了获得运动带来的满足感。体育带 来的满足感, 有利于养成良好的体育意识。 $31.2 \%$ 为了 学会技术运用, $21.8 \%$ 为了应付考试, $18.8 \%$ 为以后参与 体育活动打基础。从体育课动机上可以看出, 学生的主 观意识中还是能够认识到体育锻炼的重要性, 希望养成 体育锻炼习惯, 并且有 $35.4 \%$ 的学生对体育的兴趣有了 更高层次的需求。

\section{4. 学生喜欢的体育活动形式和内容方面满 足的要求}

调查显示, $61.5 \%$ 的学生注重身心良好体验。体育 活动过程中, 师生、学生间的身心互动较多, 比如教师 对学生进行指导、纠正动作, 学生间的相互合作、相互 帮助、相互竞争。这些互动都能给学生带来明显的情感 体验。身体锻炼和情感的双重体验是学生参加体育活动, 心理需求中较为关注的部分。54.2\%的学生希望能够摆 脱束缚, 崇尚无压力活动。体育运动能使学生产生舒适 和快感, 学生希望通过运动来释放压力、减少焦虑、消 除不良情绪, 起到调节心理的作用。体育运动带有考核 要求, 无形中让部分学生感觉到束缚和压力。 $47.9 \%$ 希 望能有不同的体验, 45. 8\%学生希望学到更多体育知识 和技能。说明这部分学生有了体育的初级意识, 对体育 有了一定的兴趣和情感, 希望对体育知识和技能有进一 步的熟知和理解, 正在向体育的中级意识迈进。29.7\% 以自己学习为主有选择的活动, $23.4 \%$ 希望开展游戏类 和竞赛类活动。

\section{5. 离开学校以后是否继续和可能从事的体 育活动}

82. 3\%的学生认为体育锻炼很重要, 即使离开学校, 也会继续进行锻炼。体育锻炼对增强体质、促进健康、 提高机体工作能力、健美形态、调节情感、减少压力方 面都有显著的作用。而且体育锻炼的形式和内容多种多 样, 学生可以根据自己将来从事的职业和身体状况, 灵 活选择项目, 进行适宜有效的锻炼。显然, 大部分学生 都能认识到体育锻炼的作用是无可替代的。相比选修课 中比较热门的三大球和小球项目, $82.8 \%$ 学生选择跑步 作为离开学校以后进行锻炼的最可行项目, 小球和三大 球分别为 $17.1 \%$ 和 $16.1 \%$, 瑜伽 $15.6 \%$, 轮滑 $13.5 \%$, 其 他 9. 3\%。跑步在实际操作中, 有效、简单、灵活。长期 坚持跑步, 能够有效控制体型, 减少脂肪, 提高人体心 肺功能。跑步对时间、场地、气候的要求相对简单。只 要你有合适的时间, 一个人也可以进行跑步。速度可快 可慢, 强度可大可小。可以根据自己的实际情况, 选择
合适的时间和方式，进行有效的锻炼。这也是跑步备受 学生推崇的体育活动的原因之一。

\section{4. 干预措施}

首先要培养学生的体育意识, 体育的兴趣和爱好是 体育的初级意识。从学生参与课外体育活动的次数上, 我们可以看出, 如果没有课外活动次数要求, 可能很多 学生都不会主动去锻炼。但是从学生认识层面上而言, 绝大部分都认为需要体育锻炼, 养成体育锻炼的习惯。 为什么有这样的认识, 却没有实际行动呢? 从调查结果 分析可以看出，学生本身对认识和对其产生的兴趣之间 有脱节。我们知道兴趣是指一个人力求认识某种事物或 从事某种活动的心理倾向。有倾向, 还需要适当引导, 使得心理倾向落实到实际行动中。要想由好奇到兴趣, 由感兴趣到长期落实到实际体育锻炼中, 需要我们在平 时的体育课教学中, 根据兴趣产生的特点和学生的实际 情况进行相应的引导。

\section{1. 增加学生对体育基础知识的了解, 是培养 体育兴趣的基础}

必要的体育知识是产生体育兴趣的基础条件, 要培 养体育兴趣, 就要有相应的知识积累 ${ }^{[5]}$, 比如欣赏一场 篮球赛，首先需要对篮球规则、篮球的基本技术、技能 有所了解。在观看比赛过程中, 注意观察队员的犯规或 违例的动作, 再看裁判的判决。通过一段时间学习和观 察, 比赛中出现的一般犯规和违例都会做出正确判断。 掌握了一定的基础知识, 能更好的欣赏篮球的美。教学 中常遇到学生对体育教学内容理解不透彻, 抓不住内在 联系, 严重影响教学效率的提高, 阻碍了教学进度计划 的正常实施。经总结发现, 往往是由于学生缺乏体育基 础理论知识所致。学校体育课教学, 增加基础理论、动 作技术结构、运动生理学和解剖学等内容的学习, 对深 入理解课的内容, 对运动技能的形成, 体育锻炼兴趣的 培养都有影响。

\section{2. 变换体育活动形式，培养直接兴趣}

利用人对事物或活动本身的外部特征产生的直接 兴趣, 激发学生对体育活动或体育课堂内容产生兴趣。 这种兴趣是学生对新事物或内容在感官上产生一种新 异的刺激。举个例子, 通常我们上新授内容, 学生的兴 趣比较浓厚, 而复习的内容, 学生的兴趣就大不如前。 直接兴趣是人对活动本身感兴趣, 要培养这种直接兴趣, 在体育课或者体育活动中, 同一个项目可以采用多种方 式, 增加趣味性, 可以适当降低难度, 增加学生的体验 感, 能激发学生参与活动, 提高学生主动了解知识的热 情和兴趣。61.5\%的学生注重身心良好体验。我们在教 
学中可以根据学生心理需求和兴趣特点, 变换教学方式 和体现形式, 增加学生的锻炼的体验度, 提高兴趣。

\section{3. 明确体育活动的目的意义, 培养间接兴趣}

上面说的直接兴趣能够给学生带来感官上的新异 刺激, 但是这样的刺激反应表现是强烈短暂的, 因此仅 仅培养学生的直接兴趣还是不够的, 要让学生对体育产 生持久的兴趣, 就需要培养其间接兴趣。间接兴趣是人 对活动的结果以及其意义有了明确认识之后所产生的 兴趣。这种兴趣产生是由于学生自身认识到活动的意义 和价值而引起的主动学习的状态, 具有持久性。例如: 在体育课堂教学中我们经常会发现, 同一堂篮球课, 在 刚开始学一个新技术的时候, 学生基本表现出相同的热 情, 当进入到相对枯燥的练习环节时, 有部分学生注意 力开始不集中, 表现出不耐烦的情绪。而部分经常参加 篮球活动的学生不仅不会感到无趣, 还会更加引起他的 兴趣。这是直接兴趣和间接兴趣的最显著区别。间接兴 趣是对活动的目的或意义感兴趣, 因而, 要调动学生参 加体育活动的积极性, 就要培养学生稳定持久的间接兴 趣。

从直接兴趣过渡到间接兴趣是有一个过程的, 这个 就是教师教授的过程, 也是需要学生通过反复枯燥的练 习才能达到掌握技术、提高技能的过程。当学生利用了 他掌握的技能获得了运动的成就感, 感受到体育运动的 魅力, 他就能对此项技能的意义产生认知, 那么这就能 使直接兴趣和间接兴趣发生迁移, 这时学生的兴趣才真 正的建立起来。

\section{4. 根据自身的兴趣特点, 培养优良的兴趣品 质}

由于每个人所处的环境、所受的教育及主体条件都 不相同, 学生的兴趣都带有个性特点, 因而要根据自身 条件进行兴趣爱好的自我培养。目前开设的体育俱乐部 和专项课教学, 充分考虑到个体兴趣差异, 让学生自主 选择感兴趣的项目。俱乐部教学中, 充分考虑到学生对 同一项目兴趣程度不同, 利用三个学期, 设计了层次递 进、难度递增的教学来满足学生的多元需求。

对 $54.2 \%$ 学生希望能够摆脱规定无压力活动的需求, 我们在开展教学过程中, 需要分配更多的时间让学生自 主学习, 全身心的投入到体育活动中, 体会体育锻炼产 生的愉悦感、满足感、成就感。同时, 学校体育工作中, 增加各级各类体育活动, 满足学生业余体育锻炼的需要。 降低体育的竞技性, 充分体现学校体育的特点, 增加趣 味性和娱乐性。多宣传竞技体育资讯, 多做竞技体育的 讲解。让学生身在体育的娱乐中, 感受竞技体育的美。 既能切身参与, 又有体育引导。让体育融入到学生的日 常生活中, 只有让学生积极参与到更多的体育活动中来, 才能对体育锻炼产生了解和愉悦感。

\section{5. 有针对性地引导将来便于开展的体育活 动项目}

在对学生离开学校以后是否继续和可能从事的体 育活动的这项调查中, $82.3 \%$ 的学生认为即使离开学校 以后继续进行体育锻炼很重要, $82.8 \%$ 学生选择跑步作 为离开学校以后进行锻炼的最可行项目。不难看出, 学 生对体育锻炼有明确的需求。目前开设的体育项目中, 三大球和小球, 尽管学生很喜欢选修这两个项目, 但不 是学生离开校园后的最佳选择。大学体育是学生接受系 统体育教育的最后一站, 跑步看似简单, 学问也很多。 比如, 长距离有氧运动前后如何热身和拉伸运动, 跑动 过程中如何有效监督自己的心率和步频，如何根据自身 情况制定适宜的运动量和运动强度。对学生认可的、将 来便于开展的体育活动项目, 学校体育教学过程中将这 一类知识及时全面地普及给学生。教师做到科学教学, 引导学生科学锻炼, 这对于学生将来持续锻炼、养成终 身体育锻炼意识有积极的作用。

\section{5. 结论}

通过研究发现, 大学生的体育锻炼意识没有被完全 激发出来。绝大部分学生希望在自主学习过程中有专业 人士指导, 能一起探讨体育方面的知识, 大部分学生在 体育获得感上有成功体验。在体育锻炼的动机上都明确 表示学会科学锻炼身体, 增强体能, 希望养成坚持锻炼 的习惯。学生的体育锻炼初级意识相对较强, 但是缺乏 必要的体育基础知识, 体育活动形式相对单一, 制约了 体育兴趣的形成。体育教学中，除了根据学生自身兴趣 特点外, 还需要有针对性地加入便于学生终身体育锻炼 的项目。

\section{REFERENCES}

[1] Heilongjiang Science:Lang.Q,(2019) The cultivation of Lifelong Physical Education consciousness in college physical education, 100-101.

[2]. Journal of Beijing Sport University: Yang.C ,Xia.D.S (2004) Research on the status quo of college students 'exercise behavior consciousness and its development countermeasures, 764-766.

[3]Youth Sports:Yang.Z.C,Zhao.X(2018) Research on improving the consciousness of lifelong physical education of vocational school students, 82 .

[4]Contemporary Sports Technology: Gong.J.R,(2019)Analysis on the cultivation of students innovative consciousness and practical ability in physical education teaching.

[5]Fujian tea : Zhang.J ,(2019) Research on the influence of campus sports culture on the formation of college students health consciousness,208-209 